\title{
Metastasizing Fibrous Histiocytoma of the Skin: A Clinicopathologic and Immunohistochemical Analysis of Three Cases
}

Louis Guillou, M.D., Sandra Gebhard, M.D., Manuel Salmeron, M.D., Jean-Michel Coindre, M.D. Institutes of Pathology of Lausanne (LG, SG) and Neuchâtel (MS), Switzerland, and Bergonié Institute and University of Bordeaux II (J-MC), Bordeaux, France

The clinicopathologic and immunohistochemical features of three metastasizing fibrous histiocytomas of the skin are presented. The first patient had a $1.3-\mathrm{cm}$ nodule in the right thigh, with right inguinal lymph node metastases 19 years later. The second patient, who had a 3-cm nodule excised from his left thigh and inguinal lymph node metastasis after 4 months, had a favorable outcome 14 years after local radiotherapy and chemotherapy. The third had a $2-\mathrm{cm}$ nodule in his neck, which recurred 16 months later. Four months later, cervical lymph node metastases were found. The patient was alive and well 26 months after initial surgery. All three primary skin tumors involved the dermis and subcutis, appeared well-delineated but nonencapsulated, were associated with some degree of epidermal hyperplasia, and showed features of aneurysmal/atypical or cellular fibrous histiocytoma. The number of mitoses ranged from 6 to 11 per 10 high-power fields. Recurrences and metastases showed morphologic features similar to primary lesions. Tumor cells were positive, at least focally, for CD 68, Ki-M1p, and Factor XIIIa, and occasionally for smooth muscle actin. Desmin, CD 34, S-100 protein, and cytokeratin stainings were negative. Primary neoplasms, recurrences, and metastases showed a Mib-1 labeling index of $10 \%$ or less.

Cellular, aneurysmal, and atypical (pseudosarcomatous) fibrous histiocytomas of the skin can metastasize, yet they often show a protracted clinical course. Risk factors for metastatic dissemination include large size, high cellularity, aneurysmal changes, marked cellular pleomorphism, high mitotic activity, tumor necrosis, and repeated local recurrences.

Copyright () 2000 by The United States and Canadian Academy of Pathology, Inc.

VOL. 13, NO. 6, P. 654, 2000 Printed in the U.S.A.

Date of acceptance: December 28, 1999.

Address reprint requests to: Dr. Louis Guillou, Institut Universitaire de Pathologie, rue du Bugnon 25, 1011 Lausanne, Suisse; e-mail: louis.guillou@chuv.hospvd.ch; fax: 41213147115.
KEY WORDS: Benign fibrous histiocytoma, Dermatofibroma, Dermatofibrosarcoma, Fibrohistiocytic tumors, Malignant fibrous histiocytoma, Metastasis, Soft tissue neoplasm.

Mod Pathol 2000;13(6):654-660

Benign fibrous histiocytoma (FH; dermatofibroma) is one of the most frequent benign mesenchymal tumors of the skin. Several distinctive variants of clinical or pathologic relevance have been described (1, 2), including cellular FH (1-3), aneurysmal (angiomatoid) FH (4), atypical (pseudosarcomatous) FH or dermatofibroma with "monster cells," palisading $\mathrm{FH}$, epithelioid $\mathrm{FH}$, atrophic $\mathrm{FH}$, ossifying FH and FH with osteoclast-like giant cells (5), cholesterotic FH associated with hyperlipoproteinemia (6), and lipidized "ankle-type" FH, which is not associated with hyperlipidemia (1). Although characterized by their potential for local recurrence, especially if incompletely excised, cellular and aneurysmal FH were not known, until recently, to be capable of metastatic dissemination. In 1995, Calonje and Fletcher (4) reported a case of aneurysmal FH of the neck, which metastasized to regional lymph nodes after repeated recurrences. In 1996, Colome-Grimmer and Evans (7) reported two patients with metastasizing cellular FH. In both cases, the tumor metastasized to regional lymph nodes and lungs, in one case after repeated recurrences. Two potential additional cases of metastasizing dermatofibroma have been found in the literature $(8,9)$.

In the present study, we analyzed the clinicopathologic and immunohistochemical features of two cellular and one aneurysmal/atypical (pseudosarcomatous) FH of the skin, which had metastasized to regional lymph nodes. Most FHs of the skin are benign lesions. However, our results indicate that in rare instances, cellular, aneurysmal, and atypical variants of cutaneous FH may metastasize and could as a consequence be considered lowgrade sarcomas. 


\section{MATERIALS AND METHODS}

Three metastasizing FHs of the skin were retrieved from the consultation files of the authors (see Acknowledgments). Clinical data and follow-up information, when available, were obtained from the referring pathologists. Tissue for light microscopic examination was fixed in $4 \%$ phosphate-buffered formalin or Bouin's fixative and embedded in paraffin; 4 - $\mu$ m-thick sections were stained with hematoxylin and eosin and, in some cases, modified Gomori reticulin and Masson trichrome. Mitosis counting was performed through a $40 \times$ lens (high power field, . $174 \mathrm{~mm}^{2}$ ).

For Cases 1 and 3, additional paraffin-embedded sections were studied with different monoclonal and polyclonal antibodies as follows: CD34 (monoclonal, clone Qbend 10, diluted 1:80; Immunotech, Marseille, France), smooth muscle actin (monoclonal, clone 1A4, diluted 1:500; Sigma Chemical Co., St Louis, MO), desmin (monoclonal, clone D33, diluted 1:40; Dakopatts, Glostrup, Denmark), S-100 protein (polyclonal rabbit, prediluted, ChemMate; Dako), cytokeratin (monoclonal, clone MNF116, diluted 1:50; Dako), CD68 (monoclonal, clone Kp-1, diluted 1:200; Dako), Ki-M1p (monoclonal, diluted 1:5000, a gift from Dr. Pierre Rudolph, Kiel, Germany [10]), and Factor XIIIa (polyclonal, diluted 1:50: Behring Diagnostika AG, Zürich, Switzerland). Immunostaining was performed according to the avidin biotin complex method of Hsu et al. (11). Tissue sections were subjected to microwave oven heating before staining. All incubation and washing steps were performed at room temperature, and diaminobenzidine was used as a chromogen. Appropriate positive and negative controls were used throughout.

\section{Case Presentations}

\section{Case 1}

A 27-year-old man presented with a $1.3-\mathrm{cm}$ bluish skin nodule on the right thigh. The lesion was removed completely with free margins, and a diagnosis of benign histiocytoma was made on microscopic examination. No additional treatment was administered. At the age of 46 , a $3-\mathrm{cm}$ painless multinodular mass that had gradually enlarged for 13 years was excised from the right inguinal region. Histologic examination showed three lymph node metastases microscopically identical to the cutaneous lesion excised 19 years earlier. Computed tomographic scans of the chest, abdomen, and pelvis performed subsequently failed to show any other metastatic deposit. Histologic reevaluation of the primary tumor showed features consistent with an aneurysmal/atypical (pseudosarcomatous) variant of benign $\mathrm{FH}$.

\section{Case 2}

A 22 year-old man presented in December 1984 with a $3-\mathrm{cm}$ cutaneous nodule of the left thigh. The lesion was removed completely with tumor-free margins and diagnosed histologically as a lowgrade malignant FH. Four months later, a left inguinal lymph node gradually increased in size as to become palpable. A lymph node dissection was performed. On microscopic examination, 1 lymph node of 10 was enlarged and showed features consistent with a metastasis from the skin tumor. Metastatic workup failed to show any other malignant deposit. The patient received radiotherapy (60 Gy) to the thigh and chemotherapy (10 courses of CYVADIC regimen associating cyclophosphamide, vincristine, doxorubicin, and deticene). He is alive and well 14 years after initial diagnosis. Of interest, the patient developed a benign $\mathrm{FH}$ on the right hand in 1996 and an atypical, mitotically active, eccrine (clear cell) hidradenoma on the right thigh in 1999.

\section{Case 3}

A 28 year-old man presented with a 2 -cm cutaneous lesion of the left side of the neck. The tumor was marginally excised and diagnosed histologically as a cellular benign $\mathrm{FH}$. It recurred 16 months later as a multinodular, $3.5 \times 1.5-\mathrm{cm}$ tumor that involved the dermis, the subcutis, and the underlying skeletal muscle. At that time, because of the precocity and large size of the recurrence combined with the presence of cellular atypia and mitoses on microscopic examination, the initial histologic diagnosis was reconsidered and changed to dermatofibrosarcoma. Four months later, the patient developed several enlarged and palpable lymph nodes in the left cervical region, justifying a lymph node dissection. Eighteen lymph nodes were examined microscopically, 4 of them (1 of which measured up to $3.5 \mathrm{~cm}$ in maximal diameter) were positive for a neoplastic process that showed morphologic features similar to those of the cutaneous lesion. Metastatic workup failed to disclose any other tumor deposit. Fifteen months after cervical dissection, the patient experienced a second local recurrence, which was widely excised with tumor-free margins. At that time, regional lymph nodes were not enlarged, and chest x-rays failed to show metastatic dissemination. At the last follow-up examination 1 month later, the patient was alive and well.

\section{Pathologic Findings}

\section{Light microscopic findings}

For Patient 1, the nonulcerated primary skin tumor showed features of an aneurysmal benign FH. The lesion was well circumscribed but nonencap- 
sulated and involved the dermis and the superficial subcutaneous tissue. The epidermis was slightly hyperplastic and separated from the underlying tumor by a 1-mm-thick grenz zone. The center of the lesion showed aneurysmal and pseudoangiosarcomatous (Fig. 1) changes, whereas, at the periphery, dermal hyalinized collagen bundles were seen either surrounded by tumor cells or entrapped in the lesion, a picture commonly seen in dermatofibromas (Fig. 2). At high-power magnification, the tumor cells showed a storiform growth pattern (Fig. 3). Tumor cell nuclei were slightly hyperchromatic and sometimes nucleolated. Hemosiderin deposits, multinucleated "Touton-type" giant cells, and unior multinucleated hemosiderophages were commonly observed in areas showing hemorrhagic and/or aneurysmal changes (Fig. 4). In the latter zones, some tumor cells showed frank nuclear atypia. There was no necrosis. The lesion was excised with tumor-free margins.

Lymph node metastases showed similar histology (Fig. 5), including the growth pattern of the tumor dissecting the collagen bundles of the lymph node capsule and the lack of necrosis. Hemorrhagic

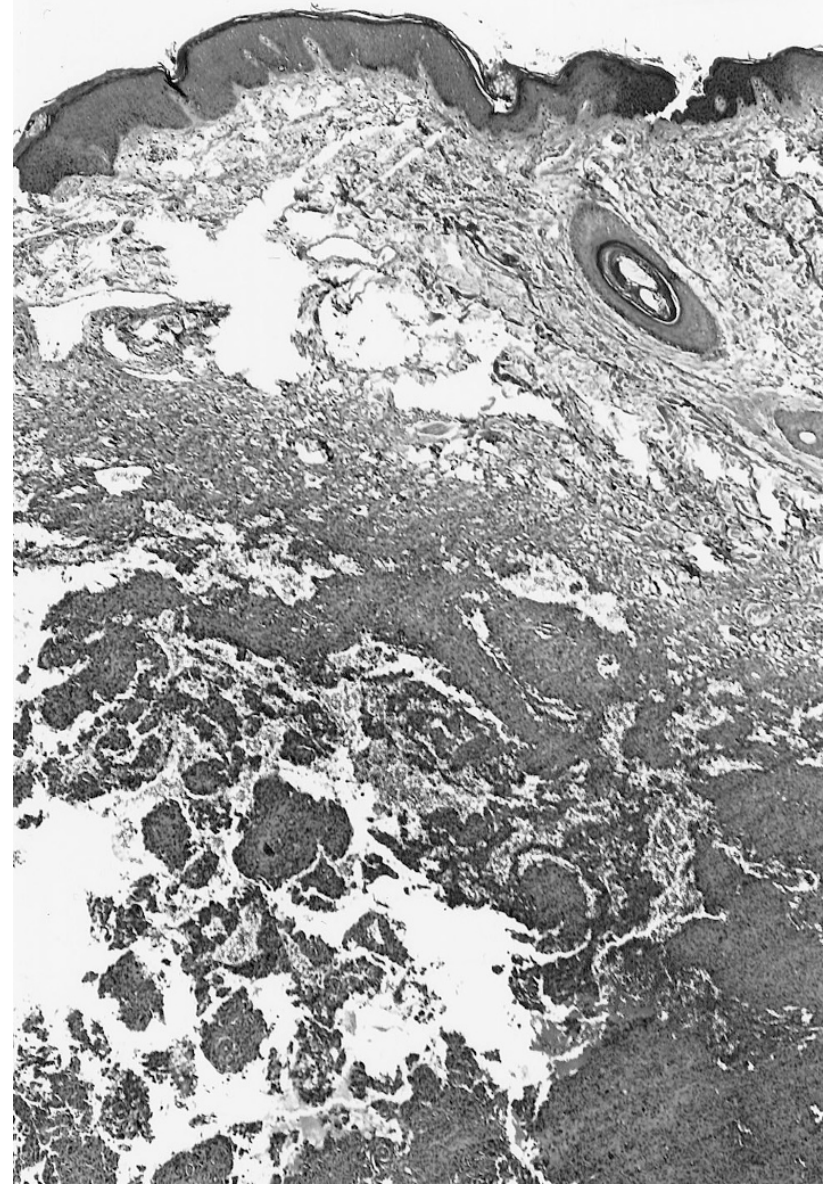

FIGURE 1. Cutaneous fibrous histiocytoma showing aneurysmal changes. The overlying epidermis is slightly hyperplastic (Case 1; hematoxylin and eosin stain; original magnification, $35 \times$ ).

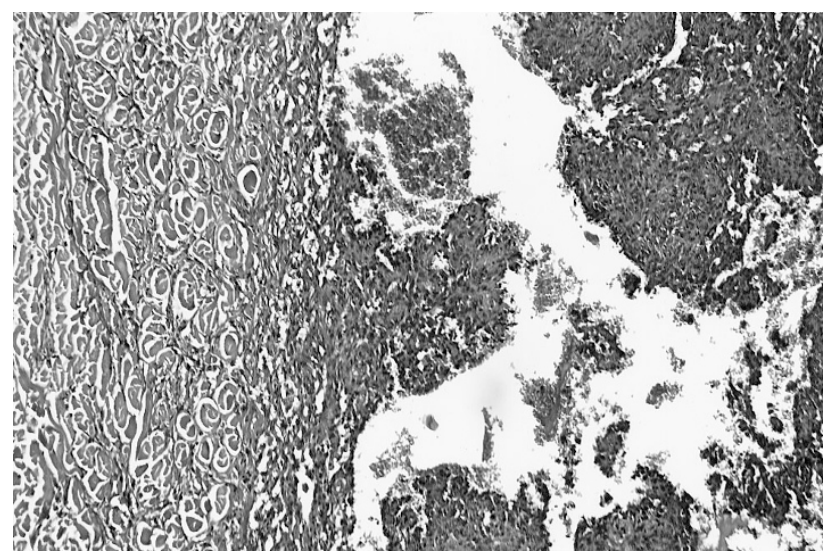

FIGURE 2. Readily visible intralesional aneurysmal changes. At the periphery, the tumor cells dissect into dermal collagen in a dermatofibroma-like manner (Case 1; hematoxylin and eosin stain; original magnification, $65 \times)$.

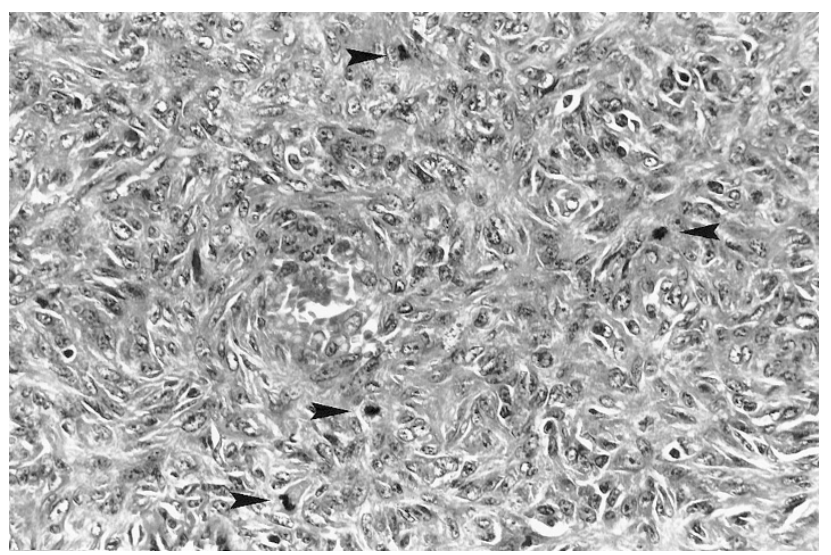

FIGURE 3. Typical storiform growth pattern with numerous mitoses (arrows) (Case 1; hematoxylin and eosin stain; original magnification, $265 \times)$.

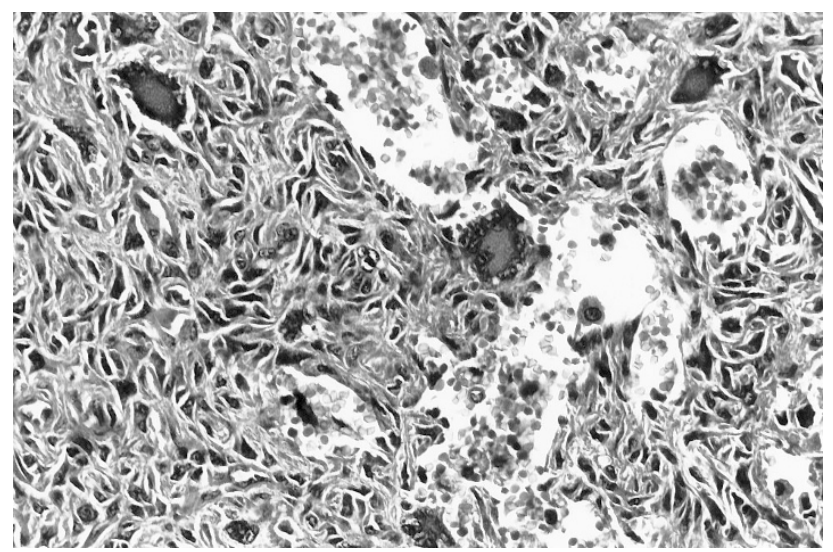

FIGURE 4. Cellular pleomorphism associated with aneurysmal changes; hemosiderin deposition; and giant, multinucleated, Toutontype siderophages (Case 1; hematoxylin and eosin stain; original magnification, $265 \times$ ).

changes and cellular atypia were less obvious (Fig. 5 , inset), and multinucleated giant cells were less numerous than in the primary lesion. The mitotic count was 8 per 10 high-power fields (hpf) in the 


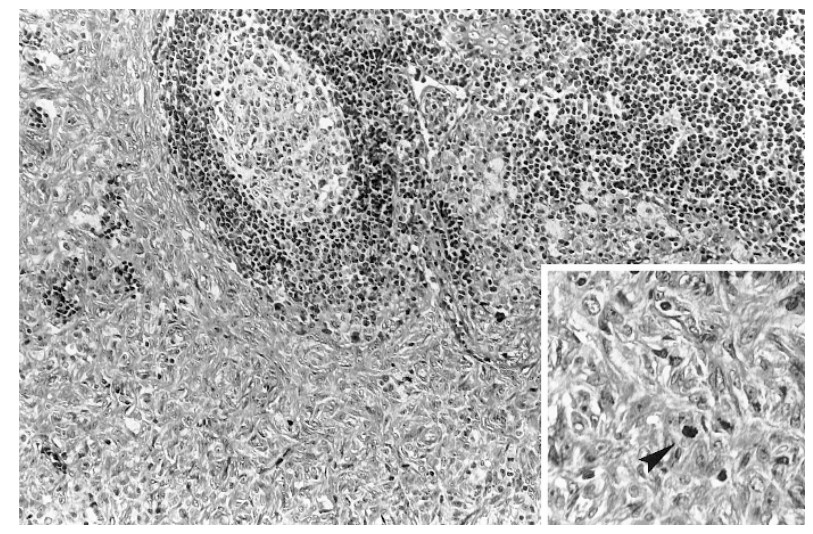

FIGURE 5. Lymph node metastasis: the cellular proliferation shows a storiform growth pattern identical to the primary lesion (Case 1; hematoxylin and eosin stain; original magnification, $130 \times$ ). Inset: cytologic atypia is minimal, and mitoses (arrow) are scarce (Case 1; hematoxylin and eosin stain; original magnification, $265 \times$ ).

original lesion and 2 per $10 \mathrm{hpf}$ in lymph node metastases.

The tumor of Patient 2 histologically presented as a cellular FH (Fig. 6). It involved the entire dermis without any residual grenz zone and extended downward into the superficial portion of the hypo-

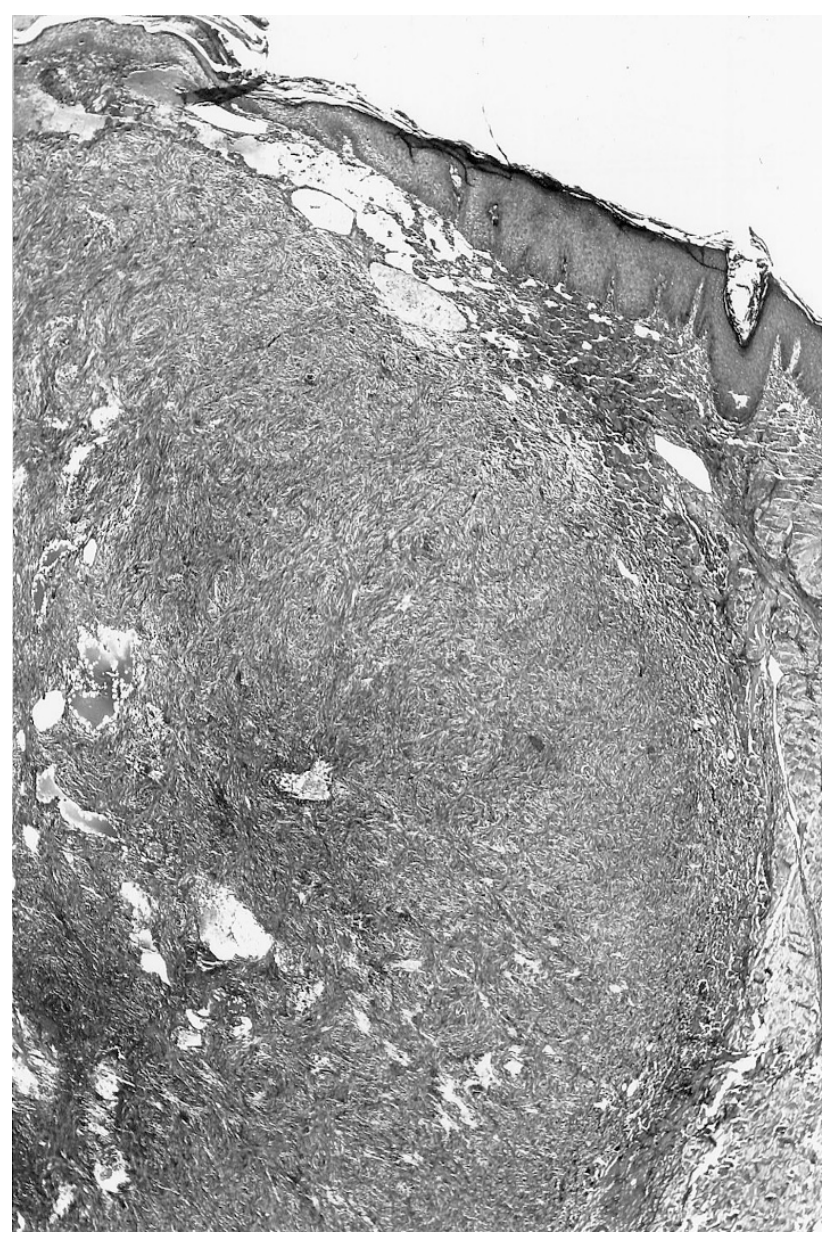

FIGURE 6. Cutaneous cellular fibrous histiocytoma extending downward to the hypodermis. The overlying epidermis is thickened (Case 2; hematoxylin and eosin stain; original magnification, $15 \times$ ). dermis. The overlying epidermis was hyperplastic but not ulcerated. Hemorrhagic and necrotic changes were observed in the center of the lesion, whereas at the periphery of the lesion, tumor cells were seen dissecting dermal collagen bundles (Fig. 7 ), together with heavy hemosiderin deposits. The mitotic count did not exceed 6 mitoses per $10 \mathrm{hpf}$, and cellular atypia was minimal, although tumor cells often showed abundant eosinophilic cytoplasm resulting in a pronounced "myoid" appearance (Fig. 7). The primary tumor was excised with tumor-free margins. Similar to the primary lesion, the tumor cells in the lymph node metastasis also had a marked myoid appearance (Fig. 8) and, focally, showed cellular disaggregation. Aneurysmal changes and necrosis were not observed. The mitotic count was 6 mitoses per $10 \mathrm{hpf}$.

For Patient 3, the primary cutaneous lesion presented as a solitary nodular mass showing typical features of cellular FH (Fig. 9). The lesion was well circumscribed, involved the entire thickness of the dermis, and encroached on the superficial hypodermal fatty tissue. The nonulcerated epidermis was slightly hyperplastic and was separated from the tumor by a 1-mm-thick grenz zone. The lesion was very cellular, composed of plumped spindle cells arranged in a typical storiform pattern (Fig. 9, inset). There was no nuclear atypia, and the mitotic count was 11/10 hpf. Tumor necrosis was observed in the center of some nodules. Hemorrhages with concomitant heavy hemosiderin deposition were also present, resembling those commonly observed in aneurysmal $\mathrm{FH}$.

The bulky recurring lesion showed a multinodular growth pattern and involved the dermis, the subcutis, and the underlying striated muscle. The lesion was separated from the epidermis by a 3-mm-thick grenz zone, and there was no concomitant epidermal hyperplasia. The proliferation was

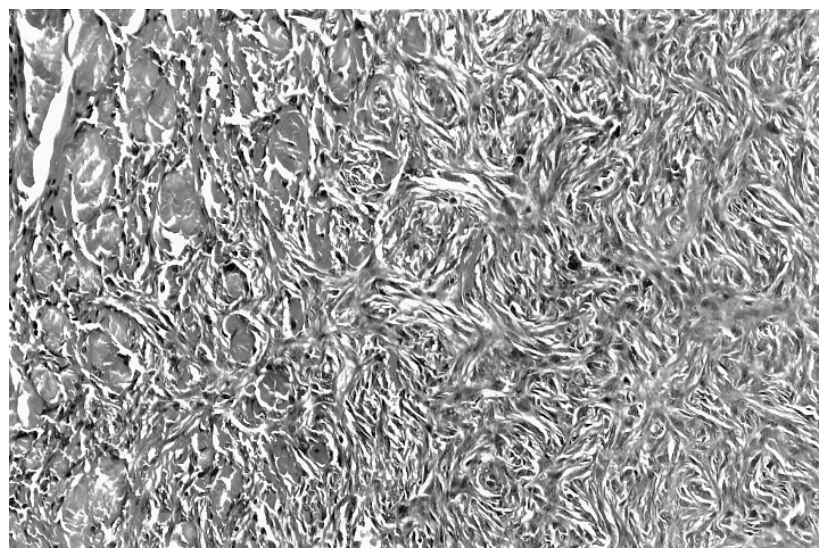

FIGURE 7. Myoid-appearing tumor cells showing a typical storiform arrangement and dissecting into dermal collagen in a dermatofibromalike manner (Case 2; hematoxylin and eosin stain; original magnification, $130 \times)$. 


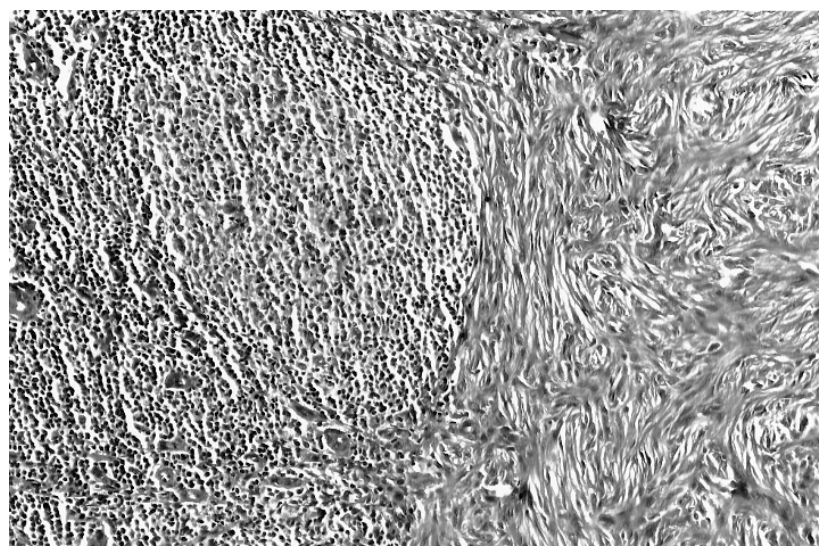

FIGURE 8. Lymph node metastasis: tumor cells are spindle shaped and have a myoid appearance, similar to that of the cutaneous tumor (Case 2; hematoxylin and eosin stain; original magnification, $130 \times$ ).

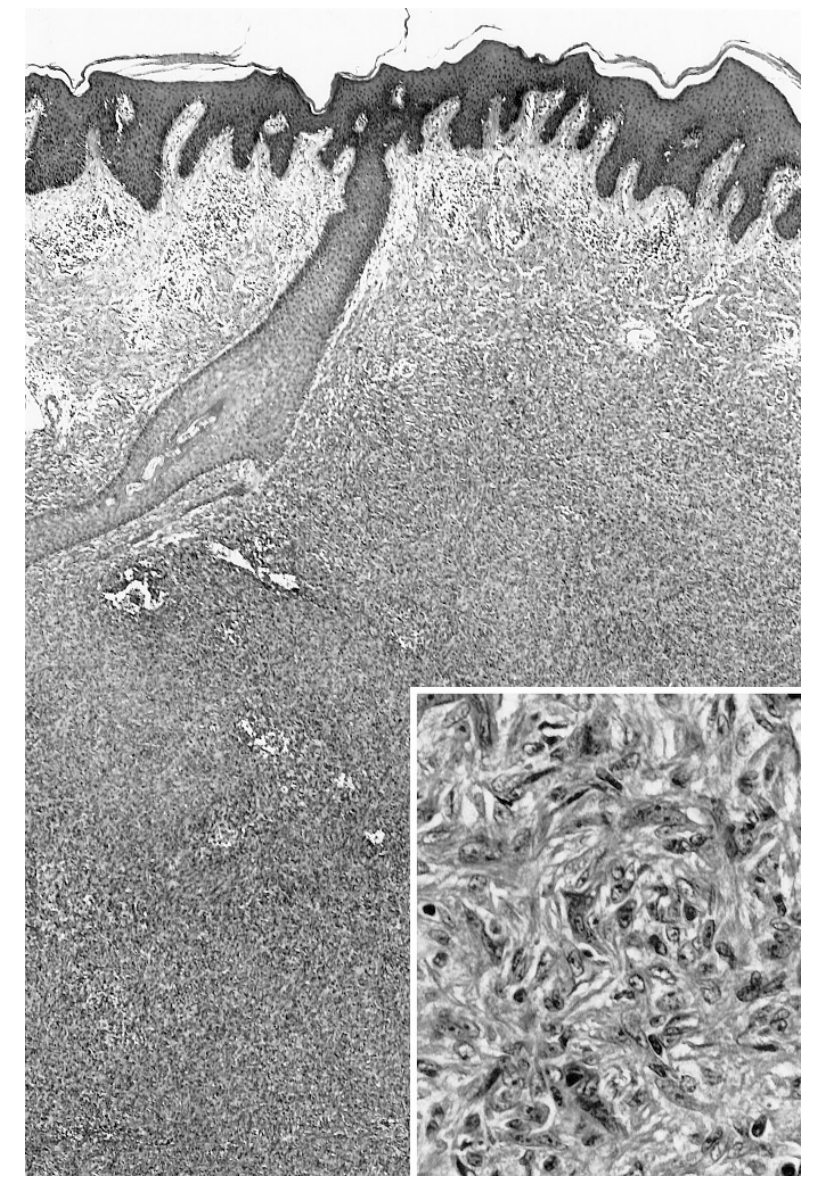

FIGURE 9. Cellular fibrous histiocytoma of the skin (Case 3; hematoxylin and eosin stain; original magnification, $25 \times$ ) showing typical storiform growth pattern (inset; hematoxylin and eosin stain; original magnification, $265 \times$ ).

very cellular, composed of plumped spindle cells arranged in a typical storiform pattern. Hemorrhagic changes with hemosiderin deposition were occasionally observed in the center of some nodules. At the edges of the lesion, the tumor cells dissected into dermal collagen, which resulted in the formation of hyalinized collagen balls often en- trapped in fields of proliferating cells. The mitotic count was 7/10 hpf. The excision specimen of the recurrence showed positive resection margins.

The lymph node metastasis showed features similar to the recurring skin tumor. The proliferating cells were arranged in either a typical storiform (Fig. 10) or a hemangiopericytoma-like pattern because of the presence of numerous branching blood vessels. The tumor cells often showed a copious myoid-like eosinophilic cytoplasm. Hemorrhages with concomitant hemosiderin deposition were focally observed. There was neither cellular atypia nor necrosis, and the number of mitoses was $3 / 10 \mathrm{hpf}$. The cells proliferated through the lymph node capsule, dissecting into the surrounding fat.

\section{Immunohistochemical findings}

In all primary tumors, recurrences, and metastases, the tumor cells were, at least focally, positive for CD68 (clone Kp-1), Factor XIIIa, and Ki-M1p, and cells in the recurrent tumor in Case 3 were focally positive for smooth muscle actin. Staining with CD34, desmin, S-100 protein, and keratin yielded negative results. The proportion of Mib-1stained nuclei never exceeded $10 \%$.

\section{DISCUSSION}

FH of the skin is a benign lesion that may recur but, until recently, was not thought no have the capacity to metastasize. In 1996, Colome-Grimmer and Evans (7) reported on two cases of cellular dermatofibroma, occurring in young adults, that metastasized to lymph nodes and lungs after repeated recurrences in one case. On microscopic examination, the primary lesions, recurrences, and metastases showed typical features of cellular dermatofibroma; lung metastases often appeared cystic. After this report, it seemed that two previously reported cases of "multiple cystic fibrohistiocytic

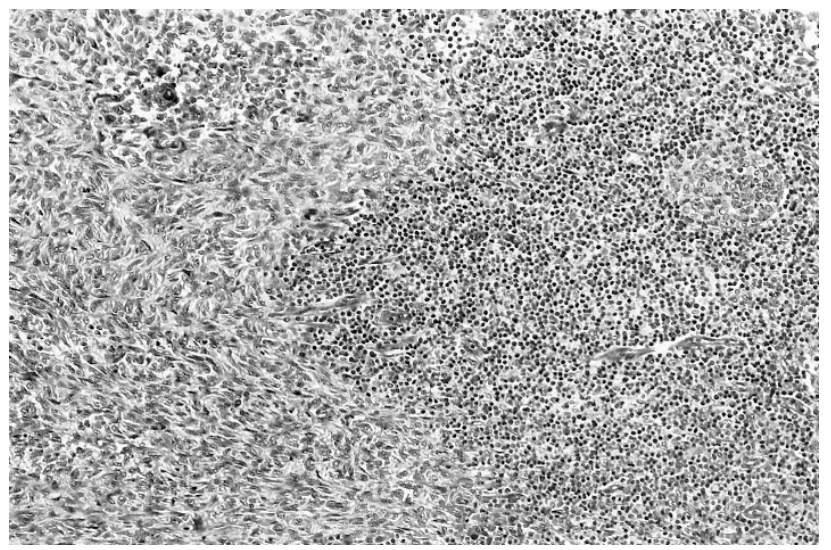

FIGURE 10. Lymph node metastasis showing storiform growth pattern (Case 3; hematoxylin and eosin stain; original magnification, $130 \times)$. 
tumors of the lung" (8) might actually have been metastatic dermatofibromas (9). In their series of aneurysmal FH, Calonje and Fletcher (4) also mentioned a case that occurred in the neck, recurred twice, and spread to a cervical lymph node. They interpreted this as a probable direct extension rather than a true metastasis.

On the basis of the present report and the observations previously published (4, 7-9) (see Table 1), it seems that some "benign" cutaneous FHs may metastasize to locoregional lymph nodes and lungs. The tumors often occur in young adults (age range, 18 to 45 years; median, 27 years); are located in the thigh (3), neck (3), and back (1); and usually present as well-demarcated, nonulcerated, bluish or brownish, relatively large (range, 1.2 to $3 \mathrm{~cm}$; median, $2 \mathrm{~cm}$ ) solitary skin nodules. The lesions metastasize preferentially to regional lymph nodes and/or lungs, often (but not always, as exemplified by two of our cases) after multiple recurrences. In most cases, there is a long delay (range, 4 to 228 months; median, 40 months) between the excision of the primary skin lesion and the emergence of metastases. Actually, long-term follow-up shows that those metastases, whatever their location, are characteristically slow-growing lesions, which justifies the iterative metastasectomy approach in case of pulmonary involvement $(7,8)$. This is exemplified by Patient 1 of our series in whom a progressive inguinal lymph node enlargement was noticed as long as 13 years before inguinal lymphadenectomy and also by Patient 2 of Colome-Grimmer and Evans's report (7), who survived 8 years after his lung metastases had been detected.

Morphologically, the primary lesions and the corresponding metastases show features characteristic of the cellular (7) (Cases 2 and 3 of the present report), aneurysmal, and/or atypical (4) (Case 1 of the present series) subtypes of FH. Characteristically, there is no increase of cellular atypia, cellular pleomorphism, mitotic activity, or proliferation indices in metastatic deposits as compared with primary lesions. There is also a strong tendency for lung metastases to undergo cystic changes (7-9), a feature that, combined with the dermatofibromalike morphology of the lesion, should lead to the correct diagnosis.

Differential diagnosis of metastasizing FH mainly includes dermatofibrosarcoma protuberans and the angiomatoid and storiform/pleomorphic subtypes of malignant FH. It is interesting that in three of five cases (7) (Case 3 of the present study), the initial diagnosis of benign $\mathrm{FH}$ was changed to angiomatoid malignant $\mathrm{FH}$ or dermatofibrosarcoma at the time of metastatic dissemination. In Case 2 of our series, the cutaneous lesion had been misdiagnosed as malignant $\mathrm{FH}$ from the beginning. The criteria that allow distinction among the cellular,

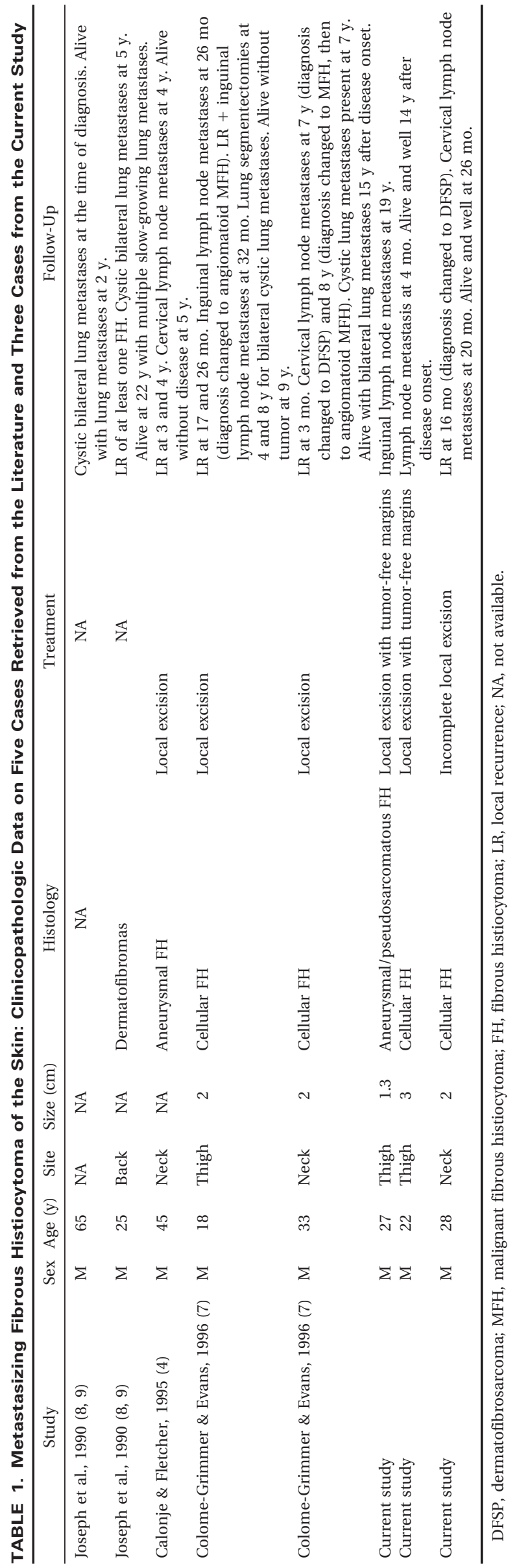

Metastasizing Cutaneous FH (L. Guillou et al.) 
aneurysmal, and atypical variants of $\mathrm{FH}$ and dermatofibrosarcoma on the one hand and malignant $\mathrm{FH}$ on the other hand have already been discussed at length $(1-4,10,12-14)$. Briefly, dermatofibrosarcoma is much less demarcated and more plaquelike and infiltrative than FH. It usually shows a more uniform cytomorphology with little atypia and few mitoses. Nuclear pleomorphism and tumor necrosis are not features of dermatofibrosarcoma in the absence of a fibrosarcomatous transformation. As opposed to cutaneous FH, most conventional dermatofibrosarcomas react with CD34 but are usually negative for Factor XIIIa, Ki-M1p, and actin (10, 12, 14). Angiomatoid malignant FH $(15,16)$ usually develops in the subcutis and presents as multinodular, cystic, and hemorrhagic masses, typically surrounded by a prominent lymphoplasmacytic infiltrate and often thick fibrous pseudocapsule. The proliferating cells are small, round to spindle shaped, and immunoreactive for desmin in approximately 50 to $80 \%$ of the cases (17). Storiform tumor growth pattern, nuclear pleomorphism and hyperchromasia, and high mitotic activity are not common features of angiomatoid FH. When located in the subcutis, the storiform/pleomorphic malignant FH generally is larger than the atypical/ pseudosarcomatous variant of benign $\mathrm{FH}$, also often multinodular, and generally shows alternating myxoid and cellular areas with obvious cellular atypia, nuclear hyperchromasia, mitoses (including abnormal mitoses), and sometimes necrosis.

We described three cases of cellular and aneurysmal/atypical cutaneous FH of the skin that metastasized to regional lymph nodes. In accordance with Colome-Grimmer and Evans (5), we believe that relatively large size, high cellularity, aneurysmal changes, marked cellular pleomorphism, high mitotic activity, tumor necrosis, and repeated local recurrences are risk factors for the development of metastases in cutaneous FH. Characteristically, those metastatic lesions show an indolent clinical course.

Acknowledgments: We thank the following pathologists and physicians for contributing case material and providing follow-up information when available: Dr. Giovannoni, Dombresson, and Prof. Tschantz, Neuchâtel, Switzerland (Case 1); Dr. Boche, Bordeaux, and Prof. Plagnol, Langon, France (Case 2); and Dr. Demol, Béthune, and Dr. Leleu, Saint-Omer, France (Case 3). We also thank Dr. Chaubert and Mrs. Burki for immunohistochemical and photographic assistance, respectively, as well as
Dr. P. Rudolph (Kiel, Germany) for having kindly provided us with the Ki-M1p antibody.

\section{REFERENCES}

1. Calonje E, Fletcher CDM. Cutaneous fibrohistiocytic tumors: an update. Adv Anat Pathol 1994;1:2-15.

2. Calonje E, Mentzel T, Fletcher CDM. Cellular benign fibrous histiocytoma. Clinicopathologic analysis of 74 cases of a distinctive variant of cutaneous fibrous histiocytoma with frequent recurrence. Am J Surg Pathol 1994;18:668-76.

3. Franquemont DW, Cooper PH, Shmookler BM, Wick MR. Benign fibrous histiocytoma of the skin with potential for local recurrence: a tumor to be distinguished from dermatofibroma. Mod Pathol 1990;3:158-63.

4. Calonje E, Fletcher CDM. Aneurysmal benign fibrous histiocytoma: clinicopathological analysis of 40 cases of a tumour frequently misdiagnosed as a vascular neoplasm. Histopathology 1995;26:323-31.

5. Kuo TT, Chan HL. Ossifying dermatofibroma with osteoclastlike giant cells. Am J Dermatopathol 1994;16:193-5.

6. Hunt SJ, Santa Cruz DJ, Miller CW. Cholesterotic fibrous histiocytoma. Its association with hyperlipoproteinemia. Arch Dermatol 1990;126:506-8.

7. Colome-Grimmer MI, Evans HL. Metastasizing cellular dermatofibroma. A report of two cases. Am J Surg Pathol 1996; 20:1361-7.

8. Joseph MG, Colby TV, Swensen SJ, Mikus JP, Gaensler EA. Multiple cystic fibrohistiocytic tumors of the lung: a report of two cases. Mayo Clin Proc 1990;65:192-7.

9. Colby TV. Metastasizing dermatofibroma. Am J Surg Pathol 1997;21:976.

10. Rudolph P, Schubert B, Wacker HH, Parwaresch R, Schubert C. Immunophenotyping of dermal spindle cell tumors: diagnostic value of monocyte marker Ki-M1p and histogenetic considerations. Am J Surg Pathol 1997;21:791-800.

11. Hsu SM, Raine L, Fanger H. Use of avidin-biotin-peroxidase complex $(\mathrm{ABC})$ in immunoperoxidase techniques: a comparison between $\mathrm{ABC}$ and unlabelled antibody (PAP) procedures. J Histochem Cytochem 1981;29:577-80.

12. Zelger B, Sidoroff A, Stanzl U, Fritsch PO, Öfner D, Zelger B, et al. Deep penetrating dermatofibroma versus dermatofibrosarcoma protuberans. A clinicopathologic comparison. Am J Surg Pathol 1994;18:677-86.

13. Kamino H, Jacobson M. Dermatofibroma extending into the subcutaneous tissue. Differential diagnosis from dermatofibrosarcoma protuberans. Am J Surg Pathol 1990;14:1156-64.

14. Mentzel T, Beham A, Katenkamp D, Dei Tos AP, Fletcher CDM. Fibrosarcomatous ("high-grade") dermatofibrosarcoma protuberans. Clinicopathologic and immunohistochemical study of a series of 41 cases with emphasis on prognostic significance. Am J Surg Pathol 1998;22:576-87.

15. Enzinger FM. Angiomatoid malignant fibrous histiocytoma. A distinct fibrohistiocytic tumor of children and young adults simulating a vascular neoplasm. Cancer 1979;44:2147-57.

16. Costa MJ, Weiss SW. Angiomatoid malignant fibrous histiocytoma. A follow-up study of 108 cases with evaluation of possible histologic predictors of outcome. Am J Surg Pathol 1990;14:1126-32.

17. Fletcher CDM. Angiomatoid "malignant fibrous histiocytoma": an immunohistochemical study indicative of myoid differentiation. Hum Pathol 1991;22:563-8. 